\title{
Radiation exposure for coronary artery calcium score at prospective 320 row multi-detector computed tomography
}

\author{
Faisal Khosa ${ }^{1}$, Atif Khan'2, Waqas Shuaib ${ }^{1}$, Melvin Clouse ${ }^{2}$, Matthew Budoff ${ }^{5}$, \\ Ron Blankstein ${ }^{4}$, Khurram Nasir ${ }^{3}$
}

\author{
${ }^{I}$ Department of Radiology, Emory University Hospital-Midtown, Atlanta, GA, USA. \\ ${ }^{2}$ Department of Radiology, Beth Israel Deaconess Medical Center, Harvard University, Boston, MA, USA. \\ ${ }^{3}$ Yale University School of Medicine, New Haven, CT, USA. \\ ${ }^{4}$ Brigham and Women's Hospital Harvard Medical School, Boston, MA, USA. \\ ${ }^{5}$ Los Angeles Biomedical Research Institute, Torrance, CA, USA.
}

Received October 16, 2013; Revised November 03, 2013; Accepted November 10, 2013; Published Online November 15, 2013

\section{Original Article}

\begin{abstract}
Purpose: To date there is extensive data on the radiation dose for assessing coronary artery calcium scores (CACS) with 4-64 row multidetector MDCT. However with the advent of 320 row MDCT, the entire heart can be imaged in one beat and thus potentially reduce the radiation dose. The aim of this study was to evaluate radiation dose for CACS on low-dose prospective EKG-triggered 320 row MDCT. Materials and Methods: Informed consent for this retrospective HIPAA-compliant study was waived and approved by our institution's institutional review board IRB. One hundred and sixty eight consecutive patients


um scoring with prospective gating. The scan parameters were $300 \mathrm{~mA}, 120 \mathrm{kVp}$, volume scan length (VSL) $160 \mathrm{~mm}$, gantry rotation $0.350 \mathrm{msec}$ and $320 \times 0.5 \mathrm{~mm}$ detectors at 320 MDCT. Beta blockers were given to patients in a case heart rate $\mathrm{HR}>65$ $\mathrm{bpm}$. The effective dose (ED) estimates were calculated for all patients from the dose length product and the conversion factor $\mathrm{k}$ $(0.014 \mathrm{mSv} / \mathrm{mGy} / \mathrm{cm})$ as recommended by current guidelines. Results: The mean SD radiation was $1.89 \pm 0.79 \mathrm{mSv}$. Overall the range varied from $0.28-2.48 \mathrm{mSv}$. The radiation was significantly less in females as compared to males $(2.02 \pm 0.73$ vs. $1.41 \pm 0.87$, $\mathrm{p}<0.0001)$. No differences were noted whether HR was $<60$ vs. $>=60 \mathrm{bpm}(1.87 \pm 0.79$ vs. $1.77 \pm 0.84 \mathrm{mSv}, \mathrm{p}=0.45)$. On the other hand a higher radiation was noted among obese individuals as compared to those with $B M I<30(1.84 \pm 0.82$ vs. $1.91 \pm 0.80 \mathrm{mSv}$, $\mathrm{p}=0.62$ ). Conclusion: Radiation dose obtained from 320-MDCT is similar to those obtained with 4-64 row MDCT. Further studies are needed to assess the feasibility of further lowering the tube current and tube voltage.
\end{abstract}

Keywords: Calcium Score; Coronary Artery; MDCT; Radiation

\section{Introduction}

Vascular calcification is a constituent of atherosclerosis and in case of coronary arteries this is a strong indicator of presence of coronary artery disease (CAD). ${ }^{1,2}$ Several studies have

Corresponding author: Waqas Shuaib, MD; Department of Radiology and Imaging Sciences, Emory University Hospital 550 Peachtree Street NE. Atlanta, GA 30308, USA.

E-mail: Waqas1184@hotmail.com

Cite this article as:

Khosa F, Khan A, Shuaib W, Clouse M, Budoff M, Blankstein R, Nasir K. Radiation exposure for coronary artery calcium score at prospective 320 row multi-detector computed tomography. Int J Cancer Ther Oncol 2013; 1(2):01023. DOI: 10.14319/ijcto.0102.3

(Dr. Faisal Khosa is an American Roentgen Ray Scholar (2013-2015))

Copyright $(\mathcal{C}$ Khosa et al. shown that the amount of coronary artery calcium (CAC) correlates with the risk for severe cardiac events, detection and quantification of CAC therefore can be a valuable diagnostic tool in the workup of patients with suspected coronary artery disease. ${ }^{3-5}$ Overall, the presence of CAC is highly sensitive and moderately specific for detection of CAD. The negative predictive value of a CAC score of zero (0) can be as high as $99 \%$ and is associated with a $0.1 \%$ annual risk of cardiovascular events ${ }^{6,7}$ and over $99 \%$ survival for 10 years. $^{8}$ $\mathrm{CAC}$ as detected and quantified using noninvasive cardiac computed tomography (CCT) represents a reliable linear anatomic estimate of total plaque burden ${ }^{9}$ and is represented clinically as a "calcium score." 
In 1990, Agatston et al. described a method to determine the amount of coronary calcium from tomographic images. ${ }^{10}$ This method depends on the area and the maximum CT density of the calcification detected by electron beam computed tomography (EBCT). EBCT has been regarded the standard of reference method for detection and quantification of coronary calcium, and studies indicating the risk for coronary artery disease are based on EBCT investigations. ${ }^{8,11,12} \mathrm{Calci}-$ um score investigations are increasingly performed by using multi-detector CT (MDCT) techniques. It has been shown that MDCT calcium scores correlate well with that of EBCT. ${ }^{13-17}$

MDCT technology has evolved with the current 320 row MDCT, offering improved coverage in the $\mathrm{z}$ axis and better temporal \& spatial resolution. These technical improvements in scanners lead to better radiation dose efficiency and improved image acquisitions of non-invasive CCTA at high quality with less radiation. The aim of this study was to evaluate radiation dose for CAC scoring on low-dose prospective EKG-triggered CCTA using 320 row MDCT.

\section{Methods and Materials}

\section{Patient Characteristics}

Informed consent for this retrospective HIPAA-compliant study was waived and approved by our institution's IRB. Retrospective review of CCTA data-base yielded 168 consecutive patients who had undergone CCTA between March 2008 and December 2009 using prospective ECG gating on 320 MDCT (Toshiba Aquilion One Dynamic volume CT, Tochigi-ken, Japan). At our institution as part of the protocol CCS is also performed on all patients. The indication for CCTA comprised the assessment of the coronary arteries or bypass grafts, chest pain, and visualization of cardiac anatomy before or after electrophysiological procedures. Typical sized patients were defined by a body mass index (BMI calculated as weight in pounds divided by height in inches) of 20-35. One hundred and sixty eight consecutive patients

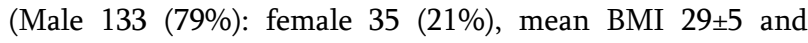
mean heart rate $58 \pm \mathrm{bpm}$ ) underwent coronary calcium scoring with prospective gating. Beta blockers were given to patients in case $\mathrm{HR}>65 \mathrm{bpm}$ (Table 1).

\section{MDCT Technique}

Aquilion ONE is a cone beam MDCT with 320 rows of 0.5 $\mathrm{mm}$ detector array capable of covering $160 \mathrm{~mm}$ of anatomy in the z-direction in one rotation without table movement. However, Aquilion ONE has two operating modes, the 64-row, and the 320-row modes. Under the 64-row mode, Aquilion ONE functions just like another 64 MDCT scanner in which both axial scans and helical scans are available with a maximum beam width (BW) of $(64 \times 0.5 \mathrm{~mm}=32 \mathrm{~mm})$.
The 320-row mode, at present, functions primarily as a cardiac scanner (volume scan mode). Under the volume scan mode, the unit acts as an axial scanner and can cover up to $160 \mathrm{~mm}$ per rotation. For cardiac scanning the BW coverage is usually 120,140 or $160 \mathrm{~mm}$ depending on the length of the heart. The tabletop translation movement is disabled via software control during image acquisition under the volume scan mode and the data set is acquired in one 360-degree rotation and image is reconstructed from 180 degrees using the remaining 180 degrees for cone beam correction.

The scan parameters during CACS measurement were 300 $\mathrm{mA}, 120 \mathrm{kVp}$, volume scan length (VSL) $160 \mathrm{~mm}$, gantry rotation $0.350 \mathrm{msec}$ and $320 \times 0.5 \mathrm{~mm}$ detectors at $320 \mathrm{MDCT}$ (Table 2).

TABLE 1: Summary of patient's characteristics

\begin{tabular}{ll}
\hline \hline Patient's Characteristics & Values \\
\hline Total subjects (n) & 168 \\
Mean Age (SD) years & $61 \pm 10$ \\
Male: Female & $133: 35$ \\
Mean BMI (SD) & $29 \pm 4$ \\
Current Smoker No/total (\%) & $13 / 168(7.7 \%)$ \\
Past smoker No/total (\%) & $74 / 168(44 \%)$ \\
Hypertension No/total (\%) & $112 / 168(66.6 \%)$ \\
Hyperlipedemia No/total (\%) & $121 / 168(72 \%)$ \\
Diabetes No/total (\%) & $23 / 168(13.7 \%)$ \\
Family H/O of CAD (\%) & $113 / 168(67.2 \%)$ \\
H/O CAD (\%) & $91 / 168(54.1 \%)$ \\
H/O of MI (\%) & $55 / 168(32.7 \%)$ \\
\hline \hline
\end{tabular}

Abbreviations: $\mathrm{SD}=$ Standard Deviation; No $=$ Number $; \mathrm{H} / \mathrm{O}=$ History of; CAD = Coronary Artery Disease; $\mathrm{MI}=$ Myocardial Infarction

TABLE 2: Scanning parameters and radiation dose for Coronary artery calcium score at 320 MDCTA

\begin{tabular}{cc}
\hline \hline $\begin{array}{c}\text { Scanning parameters \& } \\
\text { Radiation }\end{array}$ & Values \\
\hline Typical collimation & $(64 \times 5) \times 0.5 \mathrm{~mm}(160 \mathrm{~mm})$ \\
Rotation time & $350 \mathrm{sec}$ \\
Tube voltage & $120 \mathrm{kVp}$ \\
Tube current-time product & $300 \mathrm{~mA}$ \\
Scan length (mm) & $160 \mathrm{~mm}$ \\
Mean radiation dose (SD) & $1.89(0.79)$ \\
\hline \hline
\end{tabular}

Abbreviations: $\mathrm{SD}=$ Standard Deviation

\section{Radiation Dose measurement}

Radiation dose is calculated from the parameters included the volume CT dose index (CTDI vol) and dose length product (DLP). The CTDI value can be calculated as a mathematical integral under the radiation dose profile of a single rotation scan that would produce one tomographic image at a fixed table position. CTDI vol is the average radiation dose over a specific investigated volume. The dose length product (DLP) can be calculated by multiplying CTDI vol with re- 
spective scan length. DLP shows the radiation a patient is exposed to by the entire CT. This was the primary parameter in our study (Table 2).

CT dose index volume (CTDI vol) and dose length product (DLP) were recorded as direct data output from prospective ECG gated examinations. Scanner provided a protocol summary containing dose-length product for each image series. The effective radiation dose was derived from the summed dose-length product multiplied by the European working group for guidelines on Quality criteria in computed tomography conversion coefficient $(\mathrm{k}=0.014 \mathrm{mSv} / \mathrm{mGy} \mathrm{x} \mathrm{cm})$.

\section{Statistical Analysis}

The statistical software packages used for data analysis were Stata $\backslash$ MP 10.0 (Stata, college station, Tex).

\section{Results}

The mean \pm SD radiation was $1.89 \pm 0.79 \mathrm{mSv}$. Overall the range varied from $0.28-2.48 \mathrm{mSv}$. The radiation was significantly less in females as compared to males $(2.02 \pm 0.73$ vs. $1.41 \pm 0.87, \mathrm{p}<0.0001)$. No differences were noted whether HR was $<60$ vs. $>=60 \mathrm{bpm}(1.87 \pm 0.79$ vs. $1.77 \pm 0.84 \mathrm{mSv}$, p $=0.45)$. On the other hand a higher radiation was noted among obese individuals as compared to those with $\mathrm{BMI}<30$ $(1.84 \pm 0.82$ vs. $1.91 \pm 0.80 \mathrm{mSv}, \mathrm{p}=0.62)$ (Figure 1$)$.



FIG. 1: Subgroup analysis of radiation dose with reference of BMI for patients that underwent Coronary calcium score with 320 MDCTA.

\section{Discussion}

CAC scoring being a screening tool requires the use of low radiation techniques in order to outweigh the potential risks associated with the examination. A major disadvantage is the radiation exposure with the use of Computed Tomography (CT) and radiation dose has been the single most significant concern in the context of widespread clinical applications of CCTA. Medical radiation exposure has risen more than 700\% between 1980 and 2006, largely contributing to increased public awareness of medical radiation burden. ${ }^{18}$ As a reference for radiation exposure it should be noted that annually background accounts for $82 \%$, and man made for $18 \%$ of the total radiation exposure to the general population.
Amongst medical $\mathrm{x}$-rays such as diagnostic studies, computed tomography of the chest, abdomen and pelvis accounts for $58 \%$, nuclear medicine studies for $21 \%$, and consumer products for $18 \%$ of all man made exposure. CCTA contributes about $1.5 \%$ to the overall CT dose. ${ }^{18}$ Emergence of the 320 row detector CT scanner allows for even faster image acquisition for CCTA which will likely further increase the use of CCTA and be associated with a corresponding rise in radiation exposure to the public in the future. ${ }^{18}$ Calcium scoring is usually routinely performed in addition to contrast enhanced MDCTA with single rotations and prospective gating. Our measurements for the calcium score protocol at $120 \mathrm{kVp}$ compare favorably to those of prior investigations using prospective gating. ${ }^{16,19}$ Our data along with those of others also indicate that occurrence of deterministic skin effects secondary to MDCTA with the dual source and 320-detector row scanner is fundamentally inconceivable, even in the setting of performance of multiple studies in a short time span in the same individual as deterministic effects have a threshold between 2,000 and 20,000 mGy, depending on the severity. ${ }^{17}$

It is believed that transient erythema, the earliest radiation effect, is unlikely to occur with radiation doses of less than 4000-5000 mGy. ${ }^{17,} 20$ Rutten et al. reported $2.6 \mathrm{mSv}$ ED for CAC scoring with retrospectively gated 64 MDCTA but after application of maximum dose in a single phase of R-R interval and at heart rate $<60 \mathrm{bpm}, \mathrm{ED}$ was reduced from 2.6 to $1.6 \mathrm{mSv} .{ }^{21}$ While at prospectively gated 64 MDCT using step and shoot method, Malago $\mathrm{R}$ et al. and Husmann L. et al documented the ED of 1.547 and $1.1 \mathrm{mSv}$ respectively for CAC scoring. ${ }^{22,23}$ Mahnken et al. obtained a mean ED of 4.44 $\mathrm{mSv}$ (3.28-588) and $3.01 \mathrm{mSv}(2.52-4.18)$ for women and men with BMI $>26$ respectively using a four channel MDCT scanner but in comparison radiation was reduced by $11.6 \%$ for men and $24.8 \%$ for women using body weight-adapted tube current settings in case of patients with BMI below 25. ${ }^{24}$ While Hunold et al. compared the ED for electron beam and four-detector MDCT using anthropomorphic phantom. ED using electron beam CT were 1.0 and $1.3 \mathrm{mSv}$ for male and female respectively while ED were 1.5-5.2 mSv and 1.8-6.2 $\mathrm{mSv}$ for male and female respectively at 4 rows MDCT. ${ }^{25}$

We must acknowledge several limitations of our study. First, it was a retrospective review of medical records and study design was not prospective. For the retrospective study design, we reduced the bias by selecting consecutive patients. We did not assess the factors affecting the diagnostic quality of these examinations, however, the goal of this study was to assess radiation dose associated with CAC if this were to be incorporated as a screening tool to reclassify risk in patients at intermediate risk based on traditional scores such as the Framingham and Procam Algorithms. ${ }^{9}$ 


\section{Conclusion}

The 320-slice MDCT is a robust potential diagnostic tool for non-invasive coronary artery assessment with novel single rotation acquisition capabilities with single cardiac cycle. Dose optimization remains an exceedingly significant concern. Radiation dose obtained from 320-MDCT for measuring CAC is similar to those obtained with 4-64 row MDCT. Prospective studies are needed to assess the feasibility of further lowering the tube current, tube voltage, and optimizing the volume scan length to minimize radiation doses.

\section{Conflict of interest}

The authors declare that they have no conflicts of interest. The authors alone are responsible for the content and writing of the paper.

\section{References}

1. Schmermund A, Mohlenkamp S, Erbel R. Coronary artery calcium and its relationship to coronary artery disease. Cardiol Clin 2003; 21:521-534.

2. Frink RJ, Achor RW, Brown AL, Jr., Kincaid OW, Brandenburg RO. Significance of calcification of the coronary arteries. Am J Cardiol 1970; 26:241-247.

3. Guerci AD, Spadaro LA, Goodman KJ, Lledo-Perez A, Newstein D, Lerner G, et al. Comparison of electron beam computed tomography scanning and conventional risk factor assessment for the prediction of angiographic coronary artery disease. J Am Coll Cardiol 1998; 32:673-679.

4. Janssen CH, Kuijpers D, Vliegenthart R, Overbosch J, van Dijkman PR, Zijlstra F, et al. Coronary artery calcification score by multislice computed tomography predicts the outcome of dobutamine cardiovascular magnetic resonance imaging. Eur Radiol 2005; 15:1128-1134.

5. Becker CR, Majeed A, Crispin A, Knez A, Schoepf UJ, Boekstegers P, et al. CT measurement of coronary calcium mass: impact on global cardiac risk assessment. Eur Radiol 2005; 15:96-101.

6. Haberl R, Becker A, Leber A, Knez A, Becker C, Lang $C$, et al. Correlation of coronary calcification and angiographically documented stenoses in patients with suspected coronary artery disease: results of 1,764 patients. J Am Coll Cardiol 2001; 37:451-457.

7. Sarwar A, Shaw LJ, Shapiro MD, Blankstein R, Hoffman U, Cury RC, et al. Diagnostic and prognostic value of absence of coronary artery calcification. JACC Cardiovasc Imaging 2009; 2:675-688.

8. Budoff MJ, Shaw LJ, Liu ST, Weinstein SR, Mosler $\mathrm{TP}$, Tseng $\mathrm{PH}$, et al. Long-term prognosis associat- ed with coronary calcification: observations from a registry of 25,253 patients. J Am Coll Cardiol 2007; 49:1860-1870.

9. Budoff MJ, Achenbach S, Blumenthal RS, Carr JJ, Goldin JG, Greenland P, et al. Assessment of coronary artery disease by cardiac computed tomography: a scientific statement from the American Heart Association Committee on Cardiovascular Imaging and Intervention, Council on Cardiovascular Radiology and Intervention, and Committee on Cardiac Imaging, Council on Clinical Cardiology. Circulation 2006; 114:1761-1791.

10. Agatston AS, Janowitz WR, Hildner FJ, Zusmer NR, Viamonte M, Jr., Detrano R. Quantification of coronary artery calcium using ultrafast computed tomography. J Am Coll Cardiol 1990;15:827-832.

11. Arad Y, Spadaro LA, Goodman K, Newstein D, Guerci AD. Prediction of coronary events with electron beam computed tomography. J Am Coll Cardiol 2000; 36:1253-1260.

12. Shaw LJ, Raggi P, Schisterman E, Berman DS, Callister TQ. Prognostic value of cardiac risk factors and coronary artery calcium screening for all-cause mortality. Radiology 2003; 228:826-833.

13. Becker CR, Kleffel T, Crispin A, Knez A, Young J, Schoepf UJ, et al. Coronary artery calcium measurement: agreement of multirow detector and electron beam CT. AJR Am J Roentgenol 2001; 176:1295-1298.

14. Becker CR, Jakobs TF, Aydemir S, Becker A, Knez A, Schoepf UJ, et al. Helical and single-slice conventional CT versus electron beam CT for the quantification of coronary artery calcification. $A J R$ Am J Roentgenol 2000; 174:543-547.

15. Kopp AF, Ohnesorge B, Becker C, Schroder S, Heuschmid M, Kuttner A, et al. Reproducibility and accuracy of coronary calcium measurements with multi-detector row versus electron-beam CT. Radiology 2002; 225:113-119.

16. Ulzheimer S, Kalender WA. Assessment of calcium scoring performance in cardiac computed tomography. Eur Radiol 2003; 13:484-497.

17. Stanford W, Thompson BH, Burns TL, Heery SD, Burr MC. Coronary artery calcium quantification at multi-detector row helical CT versus electron-beam CT. Radiology 2004; 230:397-402.

18. Gerber TC, Carr JJ, Arai AE, Dixon RL, Ferrari VA, Gomes AS, et al. Ionizing radiation in cardiac imaging: a science advisory from the American Heart Association Committee on Cardiac Imaging of the Council on Clinical Cardiology and Committee on Cardiovascular Imaging and Intervention of the Council on Cardiovascular Radiology and Intervention. Circulation 2009; 119:1056-1065. 
19. Kim SM, Chung MJ, Lee KS, Choe YH, Yi CA, Choe BK. Coronary calcium screening using low-dose lung cancer screening: effectiveness of MDCT with retrospective reconstruction. AJR Am J Roentgenol 2008; 190:917-922.

20. Wagner LK, Eifel PJ, Geise RA. Potential biological effects following high $\mathrm{X}$-ray dose interventional procedures. J Vasc Interv Radiol 1994; 5:71-84.

21. Rutten A, Krul SP, Meijs MF, de Vos AM, Cramer MJ, Prokop M. Variability of coronary calcium scores throughout the cardiac cycle: implications for the appropriate use of electrocardiogram-dose modulation with retrospectively gated computed tomography. Invest Radiol 2008; 43:187-194.

22. Malago R, D'Onofrio M, Baglio I, Brunelli S, Tavella $\mathrm{D}$, Beltrame $\mathrm{F}$, et al. Choice strategy of different dose-saving protocols in 64-slice MDCT coronary angiography. Radiol Med 2009; 114:1196-1213.
23. Husmann L, Herzog BA, Burger IA, Buechel RR, Pazhenkottil AP, von Schulthess P, et al. Usefulness of additional coronary calcium scoring in low-dose CT coronary angiography with prospective ECG-triggering impact on total effective radiation dose and diagnostic accuracy. Acad Radiol 2010; 17:201-206.

24. Mahnken AH, Wildberger JE, Simon J, Koos R, Flohr TG, Schaller S, et al. Detection of coronary calcifications: feasibility of dose reduction with a body weight-adapted examination protocol. AJR Am J Roentgenol 2003; 181:533-538.

25. Hunold P, Vogt FM, Schmermund A, Debatin JF, Kerkhoff G, Budde $T$, et al. Radiation exposure during cardiac CT: effective doses at multi-detector row CT and electron-beam CT. Radiology 2003; 226:145-152. 